JTTEE5 18:727

DOI: 10.1007/s11666-009-9403-5

1059-9630/\$19.00 C ASM International

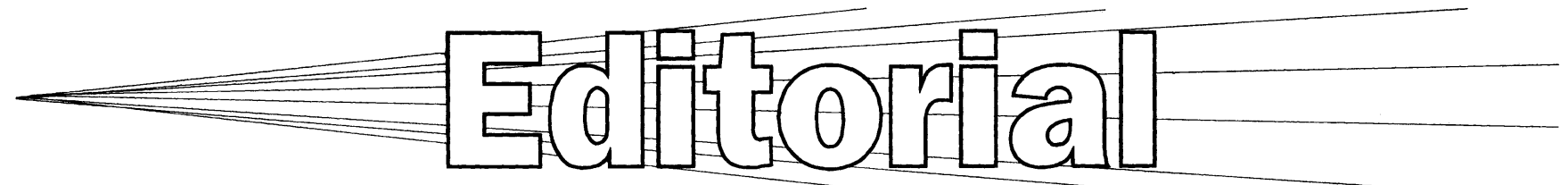

\title{
JTST Special "Recent Advances in Modeling and Simulation of Thermal Spray Processes" Issue
}

A major effort in the further development of thermal spray applications has been the improvement of process reliability and predictability in response to process changes. The ability to model a process is a prerequisite to understand and to control it. Modeling can be approached in different ways, from a qualitative or intuitive description of physical effects, or a statistical correlation of responses of coating results to changes in process parameters, to a quantitative prediction of the consequences of process parameter changes based on physical principles.

Thermal spray process modeling poses several formidable difficulties when one approaches a quantitative or predictive description of the process because of the wide range of time and length scales involved, and the various different media one has to con-

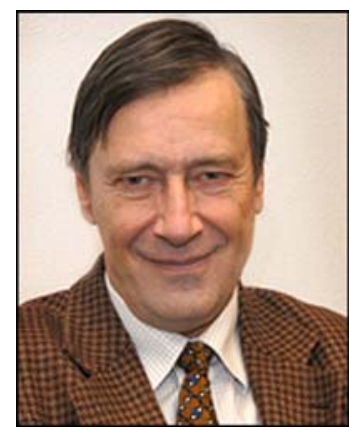

Joachim Heberlein

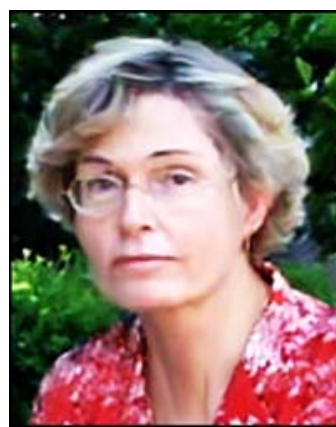

Armelle Vardelle sider. The time scales range from the splatting process that occurs in microseconds to the establishment of the coating in minutes, or even hours. Also, the media include the plasma or the combustion gases in the torch and the turbulent jet, the dispersed particles in the jet, the liquid droplet hitting the substrate, and finally the material solidifying and exhibiting various stresses. Because the various physical phenomena encountered are so different, modeling approaches have pursued the development of separate descriptions for each of the major phenomena, i.e. the plasma or hot gas generation, the plasma-particle interaction in a fluctuating jet, the splatting and coating formation, and the mechanical and thermal properties of the coatings, including the stresses. Our belief that no single review article can do justice to the variety of these phenomena has led to the publication of this issue, offering contributions by experts in the different fields.

The papers presented in this Journal of Thermal Spray Technology issue address the state of the art in many of these diverse areas of importance to thermal spraying. Moreover, the papers in this issue describe these phenomena for a variety of thermal coating processes, including plasma spraying, HVOF, cold spray, and for injection of powders and liquid precursors. Several contributions describe both the modeling approach and their validation by means of experimental work. The variety of topics in thermal spray modeling and the high quality of the contributions are likely to make this issue of JTST an invaluable reference for anyone who wishes to deepen his/her understanding of the spraying process. We expect this issue not only to serve as a yard stick and reference point for future modeling efforts, but also to influence development of specific products and processes. Lastly, because some of the modeling approaches can be generalized and transferred to other materials processing areas, we anticipate that scientists and engineers in related fields will find this information useful for their own applications.

\section{Guest Editors}

Joachim Heberlein

University of Minnesota

Minneapolis, $\mathrm{MN}$

e-mail: jvrh@me.umn.ed

Armelle Vardelle

University of Limoges

Limoges, France

e-mail: armelle@ensil.unilim.fr 